\title{
Self-Reforming Routing for Stochastic Search in VLSI Interconnection Layout
}

\author{
Yukiko KUBO $\quad$ Yasuhiro TAKASHIMA ${ }^{\dagger}$ Shigetoshi NAKATAKE ${ }^{\ddagger} \quad$ Yoji KAJITANI \\ Department of \\ Electrical and Electronic Eng. \\ Tokyo Inst. of Technology \\ 2-12-1 Ookayama, Meguro-ku, \\ Tokyo 152-8552, Japan \\ Tel: $+81-3-5734-2665$ \\ Fax: +82-3-5734-2902 \\ ${ }^{\ddagger}$ Faculty of International Environmental Eng. \\ Promotion and Development Office \\ Kitakyushu University \\ Kokuraminami-ku,Kitakyushu-city \\ Fukuoka 802-8577, Japan \\ Tel: $+81-93-964-4270$ \\ Fax: +81-93-964-4275
}

e-mail: kubo@ss.titech.ac.jp takasima@jaist.ac.jp nakatake@kitakyu-u.ac.jp

kajitani@ss.titech.ac.jp

\begin{abstract}
Given a route which connects terminal$\mathrm{s}$ on a one-layer routing area(Steiner tree), flip is a procedure that makes a current route change its configuration within its peripheral domain. A flip reforms a route by replacing one of its edges with a minimal detour. A route can flip one nearby obstacle. If the obstacle is another route, a more organized operation, called the dual flip, is applied to a pair of routes. The idea is enhanced to the 2-layer hv-routing. The performance of flip and dual flip was experimented in simulated annealing which reforms a route or a set of routes with respect to the evaluation function of multiple objectives. Some unique and satisfiable results were observed.
\end{abstract}

\section{INTRODUCTION}

Recent development of interconnection technologies enables automation tools to complete routing without much difficulties. ! !! However, it does not mean at all any solution to the difficulties due to the design environment caused by, for example, 3D-capacity, crosstalk, inductance, non-linear delay model, and other miscellanea that aggravate the yield.

Simultaneously, it is being understood that the constructive ways of routing that takes these multiple performance targets into considerations cannot be effective. There have been many studies that are successful as a single performance driven routing, such as $[2,4,5,6,7]$. But their enhanced algorithms to pursue multiple objectives are not convincing enough because the weight of different objectives is determined not based on a concrete base. As results, the routing for performance has become a more serious problem than ever.

Owing to the recent progress of computation resources, stochastic search is becoming effective. If we use this methodology, the key issue is in a quick generation of feasible routes. For the purpose, we propose a procedure, called the flip. It makes one route change its configuration within its peripheral domain. Here a route is modeled by a Steiner tree on a planar graph connecting designated terminals. By flip, a route is reformed by replacing one of its edges with a minimal detour in the sense that it flips a single nearby obstacle. It is proved that any configuaration is reachable from any configuration by a proper repetition of flips.

Some enhancements are proposed for practical use. If the obstacle is another route, a dynamical operation, called the dual-flip, is applied. For the 2-layer routing area by hv-rule, a similar operation is introduced.

An algorithm which improves the route configuration or a set of route configurations was implemented in a simulated annealing where the move is the flip or dual-flip. The evaluation was chosen from the total length, bend (via), skew, and crosstalk. The results were not only satisfiable as statics but also interesting.

Finally we note the difference of flip and well-known operation "rip-up and reroute" (abbreviated as R-R) which shows a similar behavior. See [1] for example. $R-R$ is a step in construction stage: it tries to make an unrouted connection complete at the sacrifice of other completed routes. As its nature, $R-R$ is triggered only when it meets an obstacle that blocks the routing. R-R cannot be used as a move in stochastic search. While, the flip reforms a route to adapt itself to the circumstances.

\section{Preliminaries}

In our model, a connection requirement is defined on a plane graph $G(V, E)$ such that a given set of terminals $R \subset V$ shall be connected. For simplicity of discussions, we assume that a graph is biconnected graph, that is, any pair of vertices in the graph are connected by two or more vertex disjoint paths. 
When we consider a graph which is not biconnected, we divide the graph into a set of biconnected components and consider each component independently.

The edge set that connects the terminals as specified is called a Steiner edge set. It allows any redundant edges to be included but it is called a Steiner tree if it is minimal i.e. deletion of any one edge makes it not a Steiner edge set.

Lemma 1 A Steiner edge set is a Steiner tree if and only if it contains neither cycle nor a pendant edge. A pendant edge is an edge whose one end vertex is not a terminal but of degree 1.

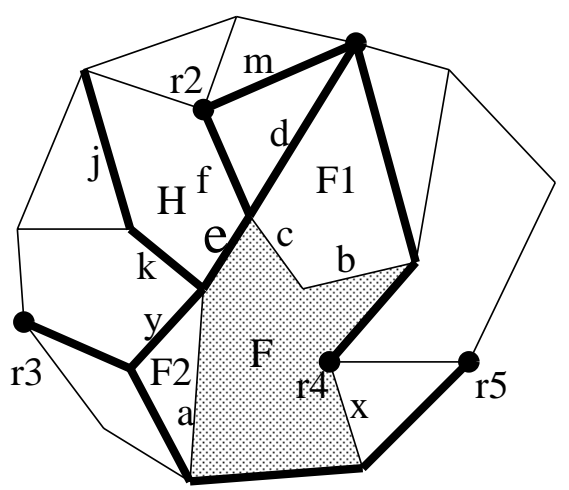

Fig. 1. Terminal set $R=\{r 1, r 2, r 3, r 4, r 5\}$, a Steiner edge set $S$, pendant edge $j$, a Steiner tree $T=S-\{j, k, m\}$, and face $F$ (shaded)

Given a Steiner edge set $S$, an operation to repeat deletion of a pendant edge or an edge in a cycle as long as a pendant edge or a cycle remains is called the reduction of a Steiner edge set $S$. In Fig.1, deletion of edges $\{j, k, m\}$, $\{j, k, d\}$ or $\{j, k, f\}$ is the reduction of $S$.

A cycle divides the plane into two regions. One is called the inner region if it is in the right-hand side traversing the cycle clock-wise, and the other the outer region.

Given a cycle, a path in the inner region that connects two distinct vertices on the cycle is called a chord path. A cycle without any chord path is called the face-cycle. The inner region of a face-cycle is the face. If a face is denoted by $F$, its corresponding face-cycle is denoted by $\bar{F}$.

An edge belongs to two face-cycles. The outer region of the graph is considered as a face. In the figure, $e$ belongs two face-cycles $\bar{F}$ and $\bar{H}$.

Lemma 2 For a Steiner tree $T$ and a face-cycle $\bar{F}$ such that $e \in T \cap \bar{F}$,

1. $T^{\prime}=T \cup \bar{F}-\{e\}$ is a Steiner edge set, and

2. reduction of $T^{\prime}$ leads a unique Steiner tree if deletion of cycle edges is restricted to those on $\bar{F}$.
In Fig.1, given $T=S-\{j, k, m\}, T^{\prime}=T \cup \bar{F}-\{e\}$ contains two face-cycles $F 1, F 2$. After the reduction as in the lemma, the resultant Steiner tree is uniquely $T^{\prime}-$ $\{a, b, c, y\}$.

\section{FLIP}

The flip is an operation which works for any 3-tuple of a Steiner tree, a face-cycle and an edge.

procedure flip $(T, e, \bar{F})$

step 1: If $e \notin T \cap \bar{F}$, output $T$. Otherwise:

step 2: $T=T \cup \bar{F}-\{e\}$

step 3: Apply the reduction to $T$ where cycle edges to be deleted is restricted to those that belong to $\bar{F}$.

An example of one-time application of flip is shown in Fig.2.

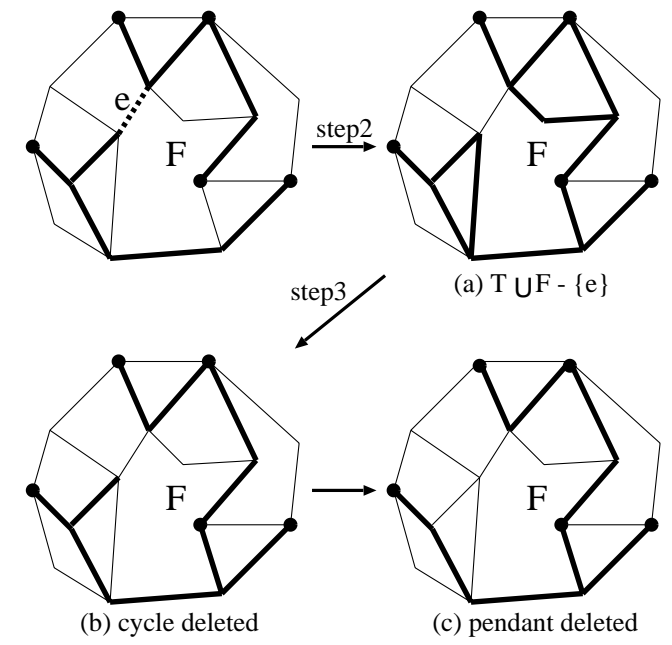

Fig. 2. Flip for input $(T, e, \bar{F})$

Theorem 1 The flip $(T, e, \bar{F})$ outputs a unique Steiner tree.

As a universal operation independent from the objective function, the reachability is crucial where a Steiner tree is said reachable from another Steiner tree if the former is obtained from the latter by repetition of proper flips.

Theorem 2 Any Steiner tree is reachable from any Steiner tree by at most $|E|-|V|$ repetitions of flips.

If we need a rigorous proof, we have to introduce some function which measures the difference between two Steiner trees. For the space, we sketch the proof taking an 
example shown in Fig.3. We are going to apply necessary flips to get $T_{b}$ from $T_{a}$.

Let $B$ denote the sum of inner regions of cycles in $T_{a} \cup$ $T_{b}$. In the figure, it is the shaded region. Let $d\left(T_{a}, T_{b}\right)$ be the number of distinct face-cycles contained in $B$. Note that $T_{a}=T_{b}$ if $d\left(T_{a}, T_{b}\right)=0$. This function is used to indicate the degree of difference between two Steiner trees. In (a), $d\left(T_{a}, T_{b}\right)=5$. Find an edge $e$ such that $e \in T_{b}-T_{a}$ and let $F$ be a face in $B$ such that $e \in \bar{F}$. Then, it is trivial that after applying $\operatorname{flip}\left(T_{a}, e, \bar{F}\right), d\left(T_{a}, T_{b}\right)$ is decreased. In the figure, $T_{a}$ in (a) is transformed to $T_{a}^{\prime}$ in (b) by $\operatorname{flip}\left(T_{a}, e, \bar{F}\right)$ with $d\left(T_{a}^{\prime}, T_{b}\right)=4$. Again $T_{a}^{\prime}$ in (b) is transformed to $T_{a}^{\prime \prime}$ as shown in (c) with $d\left(T_{a}^{\prime \prime}, T_{b}\right)=$ 2. After two times applications of flip (neglected in the figure), $d\left(T_{a}, T_{b}\right)$ becomes zero, i.e. two Steiner trees are identical.

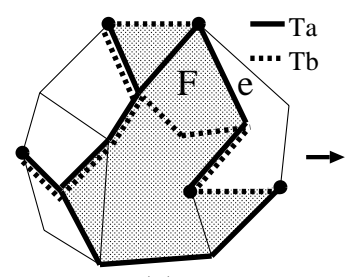

(a)

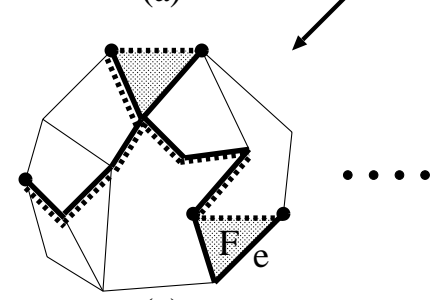

(c)
Fig. 3. Transformation of $T_{a}$ to $T_{b}$ by repetition of flips

\section{Extension OF FliP}

The model on which flip is defined is too simple. We can extend the idea in two directions, one is to handle more than one routes and the other the 2-layer routing.

\section{A. Plural Nets Optimization}

The flip is an operation which focuses a single Steiner tree where other circuit elements are equally regarded as obstacles. However, in case when the obstacle is another route, it could be reformed if it is necessary for improvement of two Steiner trees. For the purpose, a new operation, the dual-flip, is introduced.

A rough description of the dual-flip for a pair $\left(T_{a}, T_{b}\right)$ of Steiner trees is given as follows. See Fig. 4. First apply $\operatorname{flip}\left(T_{a}, e_{a} \in T_{a}, \bar{F}\right)$ to get new $T_{a}^{\prime}$ such that it has a cross on $\bar{F}$ with $T_{b}$.

Then by choosing a proper edge of $T_{b}$ and a face-cycle, apply flip $\left(T_{b}, e_{b} \in T_{b}, \bar{F}\right)$, to get $T_{b}^{\prime}$ such that $T_{a}^{\prime}$ and $T_{b}^{\prime}$ are released from crossing. This is possible if there is face-cycle $\bar{F}$ that shares a path on $T_{a}$ and that on $T_{b}$.

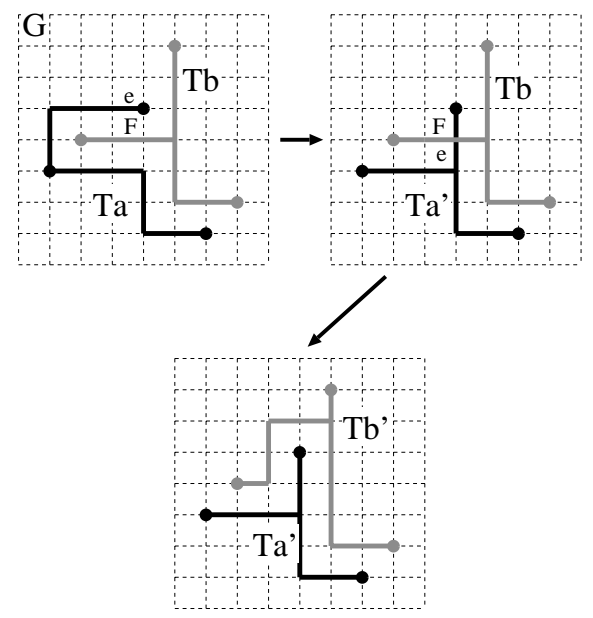

Fig. 4. Dual-flip: concatenation of flip to $T_{a}$ and flip to $T_{b}$

\section{B. General Routing Region}

The flip has been introduced assuming that the routing region or its model graph is planar. However, the concept of flip is based on two procedures "disconnect by deleting an edge" and "reconnect by a detour". Here the planarity is used only in the choice of the detour, or the face-cycle. Hence it is straightforward to extend the idea applicable to any region only if a way is fixed to choose the detour with respect to $e$.

The most important extension is to include 2-layer routing. It is easy, especially if the routing follows the hv-rule. The routing region graph is still represented by a planar grid, with an additional condition that horizontal path and vertical path can cross without fusing. Then, the minimal detour is naturally defined. An example shown in Fig.5, where deletion of edge $e$ disconnects the Steiner tree (thick line). The connection is recovered by a path that has no crossing with the obstacles.

\section{EXPERIMENTS}

We implemented procedures flip and dual flip into the algorithm:

\section{algorithm Self-Reforming Routing}

Initial: Construct an initial routing by any existing algorithm (or assume this is input).

Process: Iterate

Evaluate the solution, and keep the best so far. Update the solution by flip or dual-flip.

The algorithm is controlled by simulated annealing (SA), where parameters are decided very standard. 

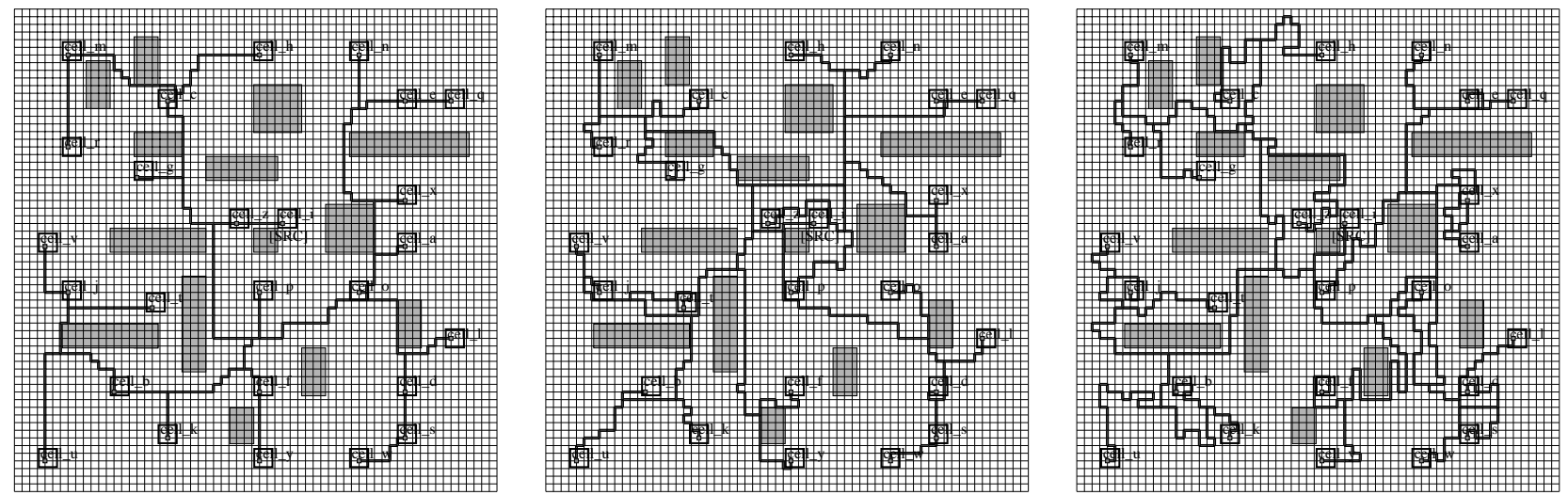

Fig. 6. Exp.A:Input tree with 25 sinks(left), a result for $\alpha=0.25$ (center) and a result for $\alpha=1$ (right)

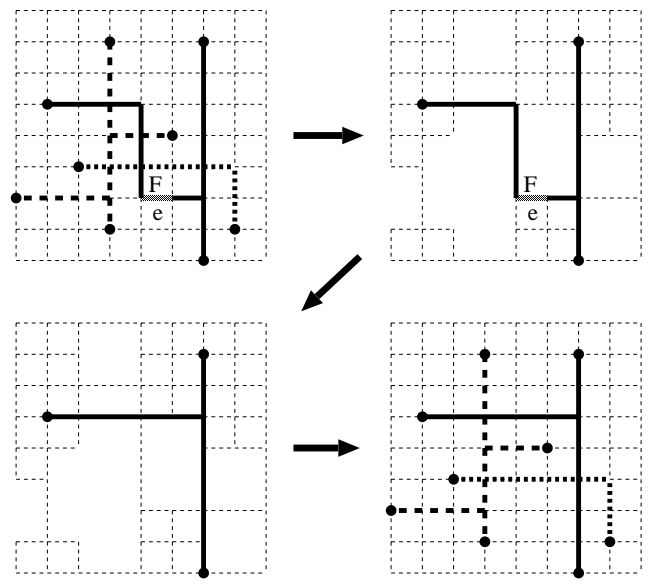

Fig. 5. 2-layer hv-routing: flip $(T, e, \bar{F})$ where the obstacle is another Steiner tree

\section{A. Clock Trees with Skew and Length Concerned}

The purpose of improving the clock tree is two folded: length and skew. We try to improve an input clock tree shown in Fig. 6(left). This is the one obtained by the Prim based maze routing. Shaded 14 rectangles in the figure are obstacles.

The move of SA is the flip. We set the evaluation function to $(L E N)+\alpha(S K E W)^{2}$, where $L E N$ is the length of the Steiner tree, $S K E W$ is the maximal skew of the length from the source to the sinks, and $\alpha$ is a parameter coefficient. If $\alpha$ is small, our algorithm seeks a Steinertree with minimal length, while if $\alpha$ is large, it tries to achieve zero-skew routing.

Experiments are for three cases of $\alpha=0,0.25$, and 1. The resultant configurations are shown in the Fig.6(center) and (right). The data is given in TABLE. I.

Observation: From TABLE.I, we could say that the algorithm is very faithful to the objective function.

A nearly zero-skew wiring is attained when $\alpha=1$. It must be noted that conventional clock-tree synthesis methods, which are $\mathrm{H}$-tree method or Deferred-merge

\begin{tabular}{|l|r|r|r|}
\hline & LEN & SKEW & time(sec) \\
\hline input tree & 1344 & 352 & - \\
\hline$\alpha=0$ & 1184 & 272 & 363 \\
\hline$\alpha=0.25$ & 1848 & 48 & 440 \\
\hline$\alpha=1$ & 2728 & 4 & 529 \\
\hline
\end{tabular}

TABLE I

EXP.A:Wire LENGTH AND MAXimAL SKEW OF A CLOCK TREE

method, are inherently unable to be applied when the region is not homogeneous by obstacles such as terminals, vias and other routes.

\section{B. Plural Steiner Trees with Length Concerned}

Suppose we are given five data each consisting of 4, 5, 6 routes as shown in TABLE.II. The configuration of input D3 is shown in Fig.7(left). The improvement request is to reduce the total length. We apply the flip and dual flip. The resultant trees are shown in Fig. 7 (center) with performance data in TABLE.III.

Observation: All the trees were equally significantly improved by around $20 \%$.

\section{Plural Steiner Trees with Multiple Objectives}

Suppose we are given three data same as D1, D2 and D3 of Exp. B as shown in TABLE.IV. They are Steiner trees obtained through the Prim based maze routing. Hence they are the results obtained by single-performance driven constructive method.

The request is to minimize following four values; LEN, BEND(number of bends), SKEW, and CTLK (crosstalk, the sum of parallel running length of two routes of distinct Steiner trees).

Let the total evaluation function be their weighted sum with coefficients L, B, S, and C, respectively.

The output Steiner-trees of D3 by weighting C are shown in Fig. 7(right).

Observation: Several noticeable issues are listed. 


\begin{tabular}{|c|c|c|c|}
\hline & \#net & \#term & LEN \\
\hline \hline D1 & 6 & 15 & 443 \\
\hline D2 & 4 & 12 & 525 \\
\hline D3 & 5 & 13 & 559 \\
\hline D4 & 5 & 15 & 498 \\
\hline D5 & 5 & 16 & 510 \\
\hline
\end{tabular}

TABLE II

EXP.B:INPUT DATA AND THE LENGTH OF INPUT TREES

\begin{tabular}{|r|r|r||r|r|}
\hline & \multicolumn{2}{|c||}{ length } & \multicolumn{2}{c|}{ time(sec) } \\
\cline { 2 - 5 } & flip only & flip \& dual & llip only & flip \& dual \\
\hline \hline D1 & 422 & 376 & 76 & 69 \\
\hline D2 & 494 & 397 & 125 & 114 \\
\hline D3 & 495 & 425 & 97 & 92 \\
\hline D4 & 438 & 398 & 78 & 73 \\
\hline D5 & 543 & 448 & 118 & 106 \\
\hline
\end{tabular}

TABLE III

EXP.B:RESULTS FOR LENGTH CONCERNED BY FLIP AND DUAL-FLIP

1. If no special weight is imposed to any performance, BEND, SKEW and CTLK are drastically improved. This is not surprising since no such attention has been given to the initial solution.

2. It is remarkable that even LEN can be improved. Remember that LEN of the input has been locally optimized.

3. The effect of weighting is apparent if we see the values on the diagonal of the table. This shows the potential of our self-reforming algorithm to respond the users.

\section{Steiner Trees on 2-Layer with Crosstalk and Vias Con- cerned}

The input is tens of routes on the 2-layer by hv-rule with respect to the crosstalk and the number of vias.

To see the average potential of our algorithm, we generate a number of input routes. One input is called class-n if it has $n$ routes. We generates 10 instances for class-30, class-40, class-50 each. One of the input is shown in Fig. 8 (left).

Before mentioning the result, some comments that may affect the results shall be added.

The input routes are the ones designed by the Prim based maze routing and then improved by reducing unnecessary vias. Though these algorithms are order dependent of the nets to be executed, we may say that they are already optimized, at least locally with respect to the length and the number of vias.

Furthermore, we checked out the route as infeasible if its length is more than 1.1 times of that of the input. This is from an experience of Experiment C, that if any length is allowed, to get a good route with respect to the crosstalk will be easy. However, such a long route is not

\begin{tabular}{|c|c|c|c|c|}
\hline & LEN & BEND & SKEW & CTLK \\
\hline \hline D1 & 443 & 116 & 53 & 97 \\
D2 & 525 & 129 & 57 & 81 \\
D3 & 559 & 150 & 65 & 122 \\
\hline
\end{tabular}

TABLE IV

EXP.C:INPUT DATA AND DATA OF INPUT TREE

\begin{tabular}{|c||c||c|c|c|c|}
\hline & L:B:S:C & LEN & BEND & SKEW & CTLK \\
\hline \hline \multirow{4}{*}{ D1 } & $1: 1: 1: 1$ & 454 & 80 & 41 & 11 \\
\cline { 2 - 6 } & $10: 1: 1: 1$ & 423 & 67 & 37 & 9 \\
\cline { 2 - 6 } & $1: 10: 1: 1$ & 442 & 59 & 53 & 127 \\
\cline { 2 - 6 } & $1: 1: 10: 1$ & 497 & 88 & 1 & 6 \\
\cline { 2 - 6 } & $1: 1: 1: 10$ & 508 & 97 & 1 & 0 \\
\hline D2 & $1: 1: 1: 1$ & 536 & 78 & 50 & 24 \\
\cline { 2 - 6 } & $10: 1: 1: 1$ & 523 & 72 & 57 & 31 \\
\cline { 2 - 6 } & $1: 10: 1: 1$ & 530 & 61 & 71 & 109 \\
\cline { 2 - 6 } & $1: 1: 10: 1$ & 642 & 148 & 15 & 34 \\
\cline { 2 - 6 } & $1: 1: 1: 10$ & 557 & 87 & 50 & 0 \\
\hline D3 & $1: 1: 1: 1$ & 678 & 99 & 1 & 56 \\
\cline { 2 - 6 } & $10: 1: 1: 1$ & 555 & 72 & 63 & 78 \\
\cline { 2 - 6 } & $1: 10: 1: 1$ & 559 & 72 & 63 & 78 \\
\cline { 2 - 6 } & $1: 1: 10: 1$ & 719 & 175 & 1 & 45 \\
\cline { 2 - 6 } & $1: 1: 1: 10$ & 698 & 119 & 1 & 1 \\
\hline
\end{tabular}

TABLE $\mathrm{V}$

EXP.C:EFFECTS OF Weighting

\begin{tabular}{|c|c|c|c|c|c|c|}
\hline & \multicolumn{2}{|c|}{ weight 0:1 } & \multicolumn{2}{c|}{ weight 1:9 } & \multicolumn{2}{c|}{ weight 1:3 } \\
\hline class & VIA & CTLK & VIA & CTLK & VIA & CTLK \\
\hline class-30 & 3.75 & 0.36 & 2.14 & 0.39 & 1.78 & 0.53 \\
\hline class-40 & 3.41 & 0.56 & 2.10 & 0.61 & 1.40 & 0.79 \\
\hline class-50 & 2.91 & 0.73 & 1.87 & 0.74 & 1.14 & 0.94 \\
\hline
\end{tabular}

TABLE VI

EXP.D:AVERAGE IMProvement OF ROUTES ON 2-LAYER

accepted as an improvement. We do not like to allow an improvement of crosstalk at the sacrifice of length.

The operation in SA is the flip.

The experiment was done three times, changing the evaluation function in the weight of the number of vias and crosstalk.

The result is given in TABLE. VI whose figures represent the ratio over the input. The result of the route given in Fig.8(left) is shown in the figure (center) and (right).

Observation: In average, in every class, the crosstalk was reduced considerably, apparently as a trade-off of the number of vias. This seems caused by the definition of the crosstalk that only exactly parallel running is counted.

\section{Conclusion}

Discussing that the search based algorithm for performance improvement of the routes is only the plausible way in recent design environment and that the key in such a technology is in generation of the candidate routes, we 

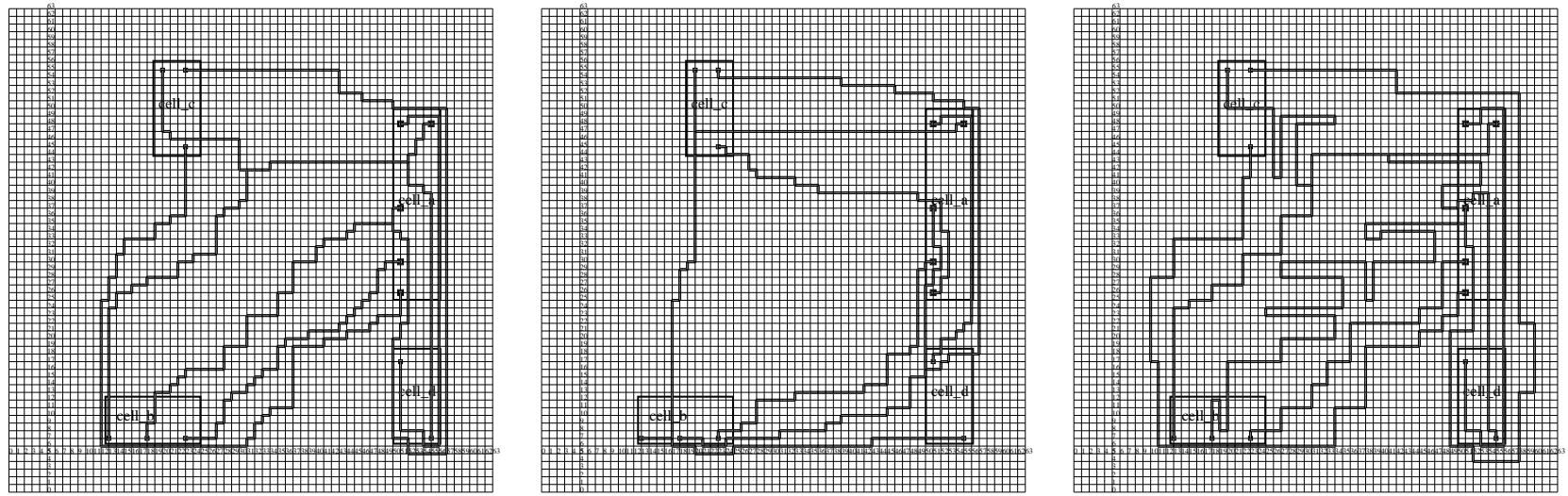

Fig. 7. Exp.B,C : Input trees of D3(left),Exp.B : length concerned(center) and Exp.C : weight ratio of 1:1:1:10(right)
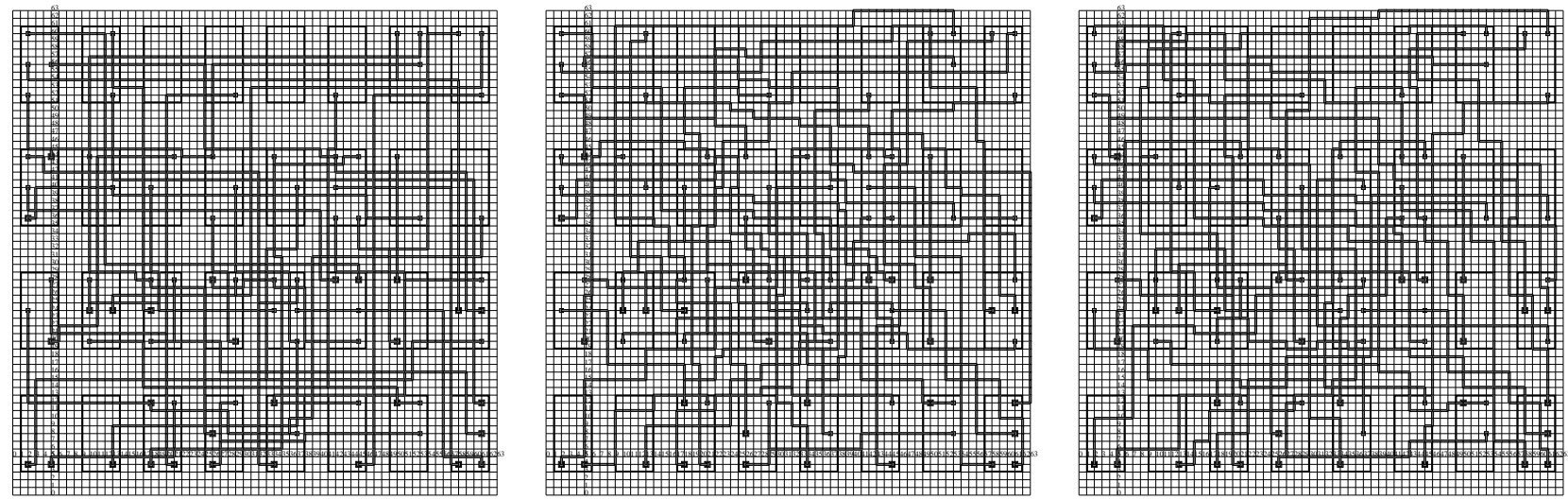

Fig. 8. Exp.D:Input trees(left), weight ratio of 1:9(center) and weight ratio of 1:3(right)

proposed the concepts of flip and dual-flip which are operations to transform the current route.

Implementing them to the SA controlled algorithm, we could show its adaptability to any performance evaluation. Since this paper is a proposal of the idea, the experiments were on the artificially made instances. For them, the experimental results showed a good performance.

Needless to say, the final objective of the work is to implement our improvement system to the practical system, where the evaluation function is so much complicated that the detailed evaluation of the performance, for example, delay of a line, can only be given by a look-up table[7], which our concept easily accepts.

\section{ACKNOWLEDGMENTS}

The authors are grateful to Associate professor Atsushi TAKAHASHI for his helpful discussion. This work is part of a project of CAD21 at Tokyo Institute of Technology.

\section{REFERENCES}

[1] H.Shin and A.Sangiovanni-V., "Mighty: A Rip-Up and Reroute Detailed Router," in Proc. ICCAD-86, pp.2-5, 1986

[2] K.D.Boese, A.B.Kahng, B.A.McCoy, and G.Robins, "Rectilinear Steiner Trees with Minimum Elmore Delay" in Proc. 31th DAC, pp.381-386, 1994.

[3] M.Edahiro, "An Efficient Zero-Skew Routing Algorithm," in Proc. 31th DAC, pp.375-380, 1994.

[4] T.Gao and C.L.Liu, "Minimum Crosstalk Channel Routing," IEEE Trans. on CAD, vol.15, no.5, pp.465-474, 1996.

[5] A.Vittal and M.M.-Sadowska, "Crosstalk Reduction for VLSI," IEEE Trans. on CAD, vol.16, no.3, pp.290-298, 1997.

[6] B.Krauter and S.Mehrotra, "Layout Based Frequency Dependent Inductance and Resistance Extraction On-Chip Interconnect Timing Analysis," in Proc. 35th DAC. pp.303-308, 1998

[7] J.Lilis and P.Buch, "Table-Lookup Methods for Improved Performance-Driven Routing," in Proc. 35th DAC. pp.368-373, 1998 